\title{
Chemisch-ökologische Zielsetzungen des Gewässerschutzes
}

\author{
Aussprache im Seenforschungslaboratorium der EAWAG/ETH \\ Kastanienbaum vom 19. bis 21. April 1972
}

\section{Zur Einführung}

Die Folgen von direkten oder indirekten Abwassereinleitungen in Binnengewässer müssen für die betroffenen Ökosysteme selbst sowie auch für die grösseren Ökosysteme der Kontinente bzw. der gesamten Erde betrachtet werden. Eine sich nur an lokalen Verhältnissen und einzelnen Gewässernutzungen orientierende Betrachtungsweise von Gewässerschutzmassnahmen ist deshalb kurzsichtig und unklug, denn sie lässt die natürlichen Bedingungen für die Erhaltung der aquatischen Ökosysteme im Rahmen ihrer vorgegebenen erdgeschichtlichen Entwicklung ausser acht. Technisch und organisatorisch besteht jedoch noch immer, trotz vieler Bemühungen, die fatale Schwierigkeit, die Folgen anthropogener Veränderungen des Zustandes eines Binnengewässers in allen Konsequenzen quantiativ zu erfassen oder gar vorauszusagen.

Das von der EAWAG angeregte Gespräch zwischen einigen Wissenschaftlern, das vom 19. bis 21. April $1972 \mathrm{im}$ Seenforschungslaboratorium der EAWAG in Kastanienbaum bei Luzern stattfand, sollte der Klärung der ökologischen Grundlagen der alten Fragen nach den Prinzipien der Gewässerbeurteilung und der numerischen Charakterisierung von Reinheitsanforderungen an eingeleitete Abwässer dienen. Einmal mehr war nach einer klaren Ausgangslage für die mit der Implementierung einer modernen Gewässerschutzgesetzgebung in der Schweiz und anderwärts verbundenen administrativen Massnahmen zu suchen. Die Realisierung eines integralen Gewässerschutzes bedingt 1. klare Vorstellungen über den gewünschten chemischen und biologischen Zustand der Gewässer eines bestimmten geographischen Raumes, 2. die Festlegung der Parameter, mit welchem Gewässerzustände unzweideutig beschrieben werden können und 3. die Aufdeckung des kausalen und quantifizierbaren Zusammenhanges zwischen Stoff-Importen in ein Gewässer und ihren ökologischen Wirkungen.

Die wesentlichen physikalischen Ökofaktoren eines Gewässers sind durch seine hydrologischen und geographischen Randbedingungen festgelegt. Die sich im Ge- 
wässer entwickelnden biologischen Systeme werden dann zur Hauptsache nur noch durch die chemischen Faktoren bestimmt, sowohl für sich allein als auch in ihrer Wechselwirkung mit den übrigen Ökofaktoren. Die grosse Dynamik der aquatischen Biosysteme ist hinsichtlich der Artenzusammensetzungen der Biozönosen und auch der Populationsdichten der vorhandenen Arten entscheidend durch die Massentransporte von Nährstoffen und Energieträgern in die Lebensräume hinein geprägt. Dies gilt sowohl makroskopisch (z.B. P-Importe in einen See) als auch für die Wechselwirkungen zwischen Organismen und Wasserchemismus im biologisch massgebenden Mikroraum um und innerhalb Organismenverbänden. Diese Transporte sind für die Zusammensetzung der Biozönosen in erster Linie bestimmend (verbreitungsgeographische Gesichtspunkte für das Auftreten oder Fehlen bestimmter Arten sind hier nicht berücksichtigt).

Die Schwierigkeiten der Beschreibung der chemisch-physikalischen Zustände der Binnengewässer haben dazu geführt, dass die biologische Phänomenologie eine dominante Bedeutung für die Analyse der Kausalität eben dieser Phänomenologie gewonnen hat - ein Zirkelschluss ohne Lösungsmöglichkeit. Nun ist aber die praktische Verwirklichung des Gewässerschutzes darauf angewiesen, Kausalketten und insbesondere ihren Anfang zu kennen. Sie muss sich zudem auf wenige gut definierte und messbare Parameter stützen können, welche Ursachen und nicht Wirkungen, d.h. Symptome, sind.

Der ursprüngliche Entschluss, die Referate sowie die zusammengefassten Diskussionen in geschlossener Form zu veröffentlichen, liess sich aus verschiedenen Gründen leider nicht in der beabsichtigten Weise realisieren. Daraufhin erklärte sich die Redaktion der Schweizerischen Zeitschrift für Hydrologie bereit, das erste Heft des Jahres 1975 für den Abdruck der Präsentationen zur Verfügung zu stellen. Die bedauerliche Verzögerung der Veröffentlichung und notwendig gewordene Kürzungen haben nach unserer Auffassung die Aktualität der Arbeit nicht beeinträchtigt. Die Veranstalter sind den Diskussionsteilnehmern dafür dankbar, dass sie sich der Mühe von teilweise erheblichen Überarbeitungen ihrer Beiträge unterzogen. 